Ralph HOFRICHTER, Pforzheim

\title{
Mathematiklernen mit digitalen Medien am Beispiel von moodle-Lernmodulen
}

Die Hochschule Pforzheim nutzt seit 2012 verstärkt Mathematik-E-Learning-Module im Learning Management System (moodle) der Hochschule.

\section{Didaktisches Konzept der moodle-Lernmodule}

Nach Kerres (2013) kann die direkte Instruktion (vgl. Leisen, 2013) auch beim Lernen mit Medien Anwendung finden und zwar vor allem bei hierarchisch strukturierten Lerninhalten, z. B. beim Erlernen von Grammatik, Mathematik, ...

Kerres beschreibt sechs Grundprinzipien der direkten Instruktion:

- Wiederholung

Der Unterricht (die Medien) beginnt mit einer kurzen Wiederholung, knüpft an bereits Gelerntes an und aktiviert Vorwissen. Lehrende und Lernende können prüfen, ob die Voraussetzungen vorliegen.

- Präsentation

Die Lehrziele werden kurz benannt. Die Lehrinhalte werden in kleinen Schritten zügig und mit Beispielen präsentiert, auf die immer Aktivitäten der Lernenden folgen. Regelmäßige Fragen prüfen, ob die Lernenden den Inhalten folgen können.

- Gestützte Übung

Die Lernenden üben alle, alleine und häufig. Sie werden vor allem am Anfang bei den Übungen betreut. Die Aufgaben werden so gestellt bzw. solange geübt, bis mindestens $80 \%$ aller Antworten richtig sind.

- Korrektur und Rückmeldung

Rückmeldungen und Verbesserungshinweise erfolgen unmittelbar.

- Selbständiges Anwenden

Es werden Lernaufgaben für selbständiges Arbeiten formuliert. Die Bearbeitung der Lernaufgaben wird kontrolliert.

- Regelmäßige Übungen und Tests

Die Lerninhalte werden auch nach bestimmten Abständen erneut bearbeitet und Angewendet.

Wichtig ist dabei, möglichst wenig Passivität aufkommen zu lassen. 
„Die direkte Instruktion zeichnet sich damit u.a. durch Präsentationen in kleinen Schritten aus, die sich regelmäßig mit Aktivitäten der Lernenden abwechseln, auf die Rückmeldungen erfolgen. ... Zentrales Element ist die Aktivität aller Lernenden!“ (Kerres, 2013)

In Anlehnung an die sechs Grundprinzipien sind der gesamte moodle-Kurs und die einzelnen Abschnitte (Lernmodule) aufgebaut. So beginnt jeder Kurs mit einem Lernmodul zur Wiederholung der Grundbegriffe. Nach jeweils kurzen inhaltlichen Präsentationen durch einen Instruktionstext mit Beispiel folgen interaktive Fragen. D. h. auf die Beantwortung der Fragen folgt unmittelbar die Rückmeldung und erforderlichenfalls eine Korrektur der gegebenen Antwort. Als Abschluss jedes einzelnen Lernmoduls erfolgt ein Test aus mehreren Fragen zum gelernten Stoff (selbständiges Anwenden), ebenfalls mit sofortiger Rückmeldung und Korrektur.

Neben dieser, für das erste Durcharbeiten empfohlenen expositorischen Methode, haben die Lernenden durch das angezeigte linke Menü jederzeit die Möglichkeit, auch eine individuelle Bearbeitungsreihenfolge zu wählen. Dadurch ist damit auch die beliebige Wiederholungsmöglichkeit der Inhalte gegeben.

\section{Integration der moodle-Lernmodule in den Mathematik-Vorkurs}

Während der stattfindenden Mathematik-Präsenz-Vorkurse haben die Studienanfänger die Möglichkeit ihre Vorkenntnisse in einem Blended-LearningSzenario zu verbessern. Grundsätzlich wechseln sich dabei seminaristische Vorlesungen im Hörsaal und Übungen mit den E-Learning-Materialien (im PC-Pool) ab. In Zweiergruppen bearbeiten die Studierenden Aufgaben aus moodle entsprechend ihres aktuellen Leistungsstandes.

\begin{tabular}{|l|l|l|l|l|l|}
\hline & Montag & Dienstag & Mittwoch & $\begin{array}{l}\text { Donners- } \\
\text { tag }\end{array}$ & Freitag \\
\hline 90 Min. & Vorlesung & Vorlesung & Vorlesung & Vorlesung & Vorlesung \\
\hline 90 Min. & Pre-Test & moodle & moodle & moodle & moodle \\
\hline 45 Min. & Vorlesung & Vorlesung & Vorlesung & Vorlesung & Vorlesung \\
\hline 90 Min. & moodle & moodle & moodle & moodle & Post-Test \\
\hline 90 Min. & Vorlesung & Vorlesung & Vorlesung & Vorlesung & \\
\hline
\end{tabular}

Typischer Plan eines Vorkurses 
In den moodle-Lernmodulen werden die wichtigsten Fragetypen verwendet (vgl. Wiegrefe, 2011), wobei auf Multiple-Choice-Fragen weitestgehend verzichtet wurde. Mit Hilfe des Plugins WIRIS (vgl. WIRIS, 2007) wurde die Mathematiktauglichkeit von moodle deutlich verbessert.

Um Lücken im Mathematik-Können zu erkennen und für den Vorkurs eine positive Motivation zu schaffen, erfolgt am ersten Tag des Kurses ein neunzig minütiger Pre-Test mit Aufgaben aus dem Grundlagenbereich (Klammern, Brüche, Rechengesetze, Gleichungen/Gleichungssysteme und Funktionen). Der Test gilt als bestanden, wenn pro Bereich mindestens 50\% der Aufgaben gelöst werden konnten. Am letzten Tag des Vorkurses wird mit einem inhaltlich vergleichbaren elektronischen Post-Test in moodle geprüft, ob eine Verbesserung der Fähigkeiten eingetreten ist.

\begin{tabular}{|l|c|c|}
\hline Semester & Bestehens-Rate Pre-Test & Bestehens-Rate Post-Test \\
\hline Sommer 2015 & $20 \%$ & $78 \%$ \\
\hline Winter 2015/16 & $28 \%$ & $87 \%$ \\
\hline Sommer 2016 & $33 \%$ & $62 \%$ \\
\hline Winter 2016/17 & $45 \%$ & $76 \%$ \\
\hline Sommer 2017 & $44 \%$ & $91 \%$ \\
\hline Winter 2017/18 & $40 \%$ & $75 \%$ \\
\hline
\end{tabular}

Bestehens-Raten Pre-Test/Post-Test der Mathematik-Vorkurse

Die Pre-/Post-Tests haben gezeigt, dass durch die Teilnahme an den Vorkursen die Leistungen in den Grundlagenbereichen deutlich besser werden. Interessant ist die Frage, welche Rolle dabei die E-Learning-Kurse spielen.

\section{Evaluation}

Von Interesse ist, wie die Studienanfänger die eingesetzten E-Learning-Materialien beurteilen. Am Ende jeden Vorkurses findet eine anonyme Befragung der Teilnehmer statt. Auf einer vierstufigen Likert-Skala von 1 (stimme voll zu) bis 4 (stimme nicht zu) soll eine Reihe von Aussagen bewertet werden. Ein Auszug aus der Evaluation des Vorkurses im Wintersemester 2017/18 (147 Teilnehmer) ist im Folgenden dargestellt: 
- Der E-Learning-Kurs hat mein Wissen vertieft. 1: $48 \% \quad 2: 40 \% \quad 3: 10 \% \quad 4: 2 \%$

- Der E-Learning-Kurs hat mir geholfen, meinen Wissensstand zu überprüfen.
$1: 70 \%$
2: $26 \%$
3: $4 \%$
4: $0 \%$

- E-Learning-Kurse sind für mich eine gute und wichtige Ergänzung zur Theorie in der Vorlesung.
1: $67 \%$
2: $30 \%$
3: $2 \%$
4: $1 \%$

\section{Wirksamkeitsstudie (MamdiM)}

Innerhalb des Projektes MamdiM (Mathematik lernen mit digitalen Medien), welches die Nutzung von interaktiven Medien beim Lernen von Mathematik beim Übergang zur Hochschule untersucht, wurden u. a. die moodle-Lernmodule der Hochschule Pforzheim untersucht. Die Konzepte von fünf Hochschulen standen im Fokus der Betrachtungen. Diese Konzepte wurden vergleichend hinsichtlich ihrer Wirksamkeit mit der Durchführung von Preund Posttests untersucht. Weiterhin standen auftretende Lern- und Kommunikationsprozesse sowie das Nutzerverhalten auf Seiten der Lernenden im Zentrum des Projekts. Erste Ergebnisse bescheinigen dem Konzept der moodle-Lernmodule eine hohe Wirksamkeit für das Entstehen einer mathematisch geprägten Kommunikation zwischen den Lernenden. Die Veröffentlichung der kompletten Studienergebnisse ist für 2018 geplant.

\section{Literatur}

Kerres, M. (2013): Mediendidaktik - Konzeption und Entwicklung mediengestützter Lernangebote, Oldenbourg Verlag München, 4. Überarbeitete und aktualisierte Auflage, München

Leisen, J. (2013): Direkte Instruktion in frontalen Unterrichtsphasen - Ein Plädoyer für die aktiv steuernde Lehrkraft, Unterricht Physik 2013, 135/136, S. $22-25$ (im Internet http://www.josefleisen.de/downloads/lehrenlernen/05\%20Direkte\%20Instruktion \%20-\%20NiU\%202013.pdf, Stand 21.6.2017)

Wiegrefe, C. (2011): Das Moodle 2 Praxisbuch - Gemeinsam online lernen in Hochschule, Schule und Unternehmen, Addison-Wesley Verlag, München

WIRIS (2007): Anleitung WIRIS 2.2, Maths for More S.L., Barcelona, verfügbar unter http://www.wiris.com/en/cas/docs/manual-pdf (Stand 27.6.17) 